\title{
PANCREAS
}

\section{Persistent organ failure during the first week as a marker of fatal outcome in acute pancreatitis}

\author{
C D Johnson, M Abu-Hilal, Members of the British Acute Pancreatitis Study Group
}

Gut 2004;53:1340-1344. doi: 10.1136/gut.2004.039883 See end of article for
authors' affiliations

Correspondence to: MrCD Johnson, University Surgical Unit, F Level, Centre Block (816), Southampton General Hospital, Southampton SO16 6YD, UK; c.d.johnson@ soton.ac.uk

Revised version received 4 March 2004

Accepted for publication

17 March 2004
Background: In predicted severe acute pancreatitis, many patients develop organ failure and recover without local complications, and mortality is only 14-30\%. It has been suggested that half of patients with progressive early organ failure may die, but there are no data to relate death or local complications to duration of early (week 1) organ failure.

Aims: To determine mortality rates in patients with transient ( $<\mathbf{4 8}$ hours) and persistent ( $>\mathbf{4 8}$ hours) early organ failure and to show whether persistent organ failure predicts death or local complications.

Patients: A total of 290 patients with predicted severe acute pancreatitis previously studied in a trial of lexipafant, recruited from 78 hospitals through 18 centres in the UK.

Method: Manual review of trial database to determine: the presence of organ failure (Marshall score $\geqslant 2$ ) on each of the first seven days in hospital, duration of organ failure, and outcome of pancreatitis (death, complications by Atlanta criteria).

Results: Early organ failure was present in $174(60 \%)$ patients. After transient organ failure $(n=71)$, outcome was good: one death and $29 \%$ local complications. Persistent organ failure $(n=103)$ was followed by 36 deaths and $77 \%$ local complications, irrespective of onset of organ failure on admission or later during the first week.

Conclusion: Duration of organ failure during the first week of predicted severe acute pancreatitis is strongly associated with the risk of death or local complications. Resolution of organ failure within 48 hours suggests a good prognosis; persistent organ failure is a marker for subsequent death or local complications.
A cute pancreatitis is a disease with a very varied outcome, ranging from complete resolution to death. Overall, the mortality rate in the UK is $7.5-9 \%^{12}$; in patients with predicted severe disease, the mortality rate ranges from $14 \%$ to $30 \%{ }^{2-4}$ depending on the criteria for prediction, and in patients with complications (severe acute pancreatitis, as defined in the Atlanta criteria ${ }^{5}$ ) the mortality rate is $25-30 \% .{ }^{4}{ }^{6}$ Approximately one third to half the deaths in acute pancreatitis occur during the first week, as a result of progressive organ failure (multiple organ dysfunction syndrome (MODS)). ${ }^{17}$ Late deaths occurring more than one week after admission to hospital are often associated with local complications, such as infected pancreatic necrosis, and these patients also usually show features of sepsis and MODS. ${ }^{7-9}$

In the past, patients with acute pancreatitis have been categorised according to the presence or absence of complications, as the definition of severe disease. This was codified in the Atlanta criteria ${ }^{5}$ which define severe pancreatitis as the presence of any complication. These complications include systemic organ failures, as well as local manifestations. However, clinical experience is that many patients with early organ failure respond rapidly to treatment and appear to have an otherwise uncomplicated outcome. Recently, it has been shown that organ failure in the first week of admission is a dynamic process and that progression of early organ failure was attended by a mortality rate in excess of $50 \%{ }^{6}$

The present study was conducted using a large database of patients with predicted severe acute pancreatitis. The aim was to categorise patients as having transient or persistent organ failure in the first week of acute pancreatitis, to investigate the relationship of these categories with fatal outcome, and to determine the relationship of persistent organ failure with subsequent development of local complications.

\section{METHOD}

This study reviews a database of patients with predicted severe acute pancreatitis entered into a placebo controlled trial of lexipafant. ${ }^{10}$ The database contained 290 patients with a confirmed diagnosis of acute pancreatitis, aged over 18 and less than 80 years, with an APACHE-II score ${ }^{11}>6$ in the 24 hours before entry to the study. All patients were primary admissions to hospital and had symptoms for less than 72 hours before entry to the study. Patients were recruited from 78 hospitals, including 18 centres constituting the British Acute Pancreatitis Study Group. All data were recorded prospectively. Patients received an infusion of either lexipafant or placebo for up to seven days and were followed until hospital discharge or death. The management of these patients was otherwise according to the usual practice of the responsible clinician.

The variables recorded in the study proformas included the presence and severity of organ failure on every day during the first week, subsequent local complications, ${ }^{5}$ subsequent episodes of sepsis, and death during the same hospital admission. Death at a later time during the trial was also recorded if notified to the researcher. The electronic database is no longer accessible to the investigators because it was held by a company that is no longer trading: this study was conducted by the manual inspection of the full version of the trial report prepared by the trial sponsor (British Biotech Plc) and provided to each member of the study group.

\section{Definition of organ failure}

Organ failure was defined as in the previous report, ${ }^{10}$ using the Marshall score, ${ }^{12}$ which approximates very closely to the definitions used in the Atlanta criteria. ${ }^{5}$ Organ failure scores

Abbreviations: MODS, multiple organ dysfunction syndrome; $C T$, computed tomography 
were recorded each day during the first week for all organ/ systems (with the exception of the hepatic score which was excluded to avoid confounding by the effects of gall stones and obstructive jaundice). A score of 2 or greater for any one organ or system equates to organ failure and was achieved when the following cut offs were exceeded: cardiovascular failure if systolic blood pressure was $\leqslant 90 \mathrm{~mm} \mathrm{Hg}$ despite fluid replacement; respiratory failure if the $\mathrm{PaO}_{2} / \mathrm{FiO}_{2}$ ratio was $<300 \mathrm{~mm} \mathrm{Hg}$; central nervous system failure if the Glasgow coma score was $<13$; coagulopathy if the platelet count was $\leqslant 80 \times 10^{9} / \mathrm{l}$; and renal failure if plasma creatinine was $>170 \mathrm{mmol} / \mathrm{l}$. If any of these criteria were met, the patient was recorded as having organ failure on that day. Transient organ failure was defined as organ failure present on less than three consecutive days excluding day 0 , and persistent organ failure was recorded when organ failure was present on three or more days after day 0 , where day 0 was the day of entry to the study and day 1 was the 24 hour period starting at 8.00am on the day after entry.

Records were inspected to determine the presence or absence of organ failure on each day of the first week of study and whether this organ failure was persistent ( $>48$ hours) or transient ( $<48$ hours). Cross tabulations were made with outcome, in particular with death and local complications. Also, records of all serious adverse events reported in the trial were inspected to gain information about complications of acute pancreatitis.

Where appropriate, differences in distribution were tested with the $\chi^{2}$ test or Fisher's exact test. Significance was accepted at the $5 \%$ level.

\section{RESULTS}

The database contained information on 290 patients of whom 40 died. Table 1 shows that a fatal outcome was almost always associated with an APACHE-II score of $>8$. Only two patients with initial APACHE-II scores of 7 and 8 died, one within 24 hours from rapidly progressive organ failure and one on day 42 from pancreatic necrosis. Patients with an APACHE-II score $>8$ on admission had an $18 \%$ mortality rate.

Of the 40 deaths, 15 occurred within the first week. All of these patients had organ failure from the day of admission until the day of death. Twenty five patients died more than one week (median 20.5 days (range 8-132)) after admission. Three late deaths at 114, 115, and 169 days were found not to be due to acute pancreatitis; the remaining 37 deaths were ascribed to complications of acute pancreatitis.

The relationship between the presence and duration of organ failure within the first week of study and subsequent death is shown in table 2. Persistent organ failure, whether present at admission or arising during the first week, was significantly associated with a fatal outcome. Of the 40 patients who died, three had no organ failure in the first week, 12 had single organ failure, and 26 had two or more early organ failures (table 3). All patients with multiple organ failures had pulmonary or renal insufficiency. Of the three patients without organ failure during the first week who

Table 1 APACHE-II scores in the first 24 hours and subsequent survival in 290 patients with predicted severe acute pancreatitis

\begin{tabular}{lcc}
\hline APACHE-II score & Survived & Died \\
\hline $4-6$ & 4 & 0 \\
7,8 & 72 & $2(3 \%)$ \\
$>8$ & 172 & $38(18 \%)$ \\
Missing data & 2 & \\
\hline
\end{tabular}

Table 2 Relationship between the presence or absence, and duration of organ failure (OF) in the first week of the study, and subsequent death in 290 patients with predicted severe acute pancreatitis

\begin{tabular}{llcc}
\hline & Survived & Died & Total \\
\hline No organ failure & 113 & 3 & 116 \\
OF at entry & 59 & 1 & 60 \\
$\quad$ Transient & 56 & 32 & 88 \\
Persistent & 11 & 0 & 11 \\
New OF within 7 days & 11 & 4 & 15 \\
$\quad$ Transient & & & \\
Persistent & &
\end{tabular}

Significant association of death with persistent organ failure compared with transient or no organ failure: $\chi^{2}=62.54 ; \mathrm{df}=2 ; \mathrm{p}<0.001$.

Table 3 Distribution of organ failures (OF) in the first week in 40 patients with acute pancreatitis who subsequently died

\begin{tabular}{llr}
\hline No organ failure & & 3 \\
Single OF & Pulmonary & 10 \\
\multirow{3}{*}{ Multiple OF } & Renal & 2 \\
& Pulmonary and renal & 12 \\
& 3 or more systems & 13 \\
\hline
\end{tabular}

Table 4 Types of organ failure recorded in 71 patients with transient ( $<48$ hours) organ failure (OF) in the first week of study

\begin{tabular}{lc}
\hline Pulmonary & 61 \\
Renal & 3 \\
Coagulopathy & 3 \\
Multiple OF & 3 \\
Pulmonary and renal & 1 \\
\hline Pulmonary and coagulopathy &
\end{tabular}

subsequently died, one developed continuing signs of sepsis with abdominal discomfort and died from presumed infected pancreatic necrosis on the 25th day. Two others died of unrelated causes (brain stem stroke at 144 days and myocardial infarction on day 5, no autopsy evidence of pancreatitis). One patient died after early organ failure that resolved within less than 48 hours. After a prolonged hospital stay, he developed pancreatic necrosis; necrosectomy was performed but his condition deteriorated and he died after 81 days from sepsis and acute renal failure.

As noted previously, ${ }^{10} 44 \%$ of patients already had organ failure at entry to the study. In the present analysis, organ failure was present in $148(51 \%)$ patients in the first 24 hours of the study (that is, on day 0 (study entry) or day 1 ). Of 174 patients who developed organ failure at entry or within the first week of the study, 71 (41\%) had transient organ failure that resolved in 48 hours or less. Of these patients only one died, from the effects of pancreatic necrosis. The types of organ failure present in patients with transient organ failure are shown in table 4 . Of the 103 patients with persistent organ failure, 36 (35\%) died, of whom 14 died within the first week from progressive organ failure.

Of 174 patients with organ failure during the first week of the study, 59 later developed local complications. In contrast, only 12 patients developed symptomatic local complications in the absence of early organ failure. Detailed analysis of the whole group was not possible because the trial protocol did 
Table 5 Relationship between duration of organ failure and development of local complications in 110 patients with record of a serious adverse event

\begin{tabular}{|c|c|c|c|c|}
\hline & $\mathbf{n}$ & $\begin{array}{l}\text { Local } \\
\text { complications }\end{array}$ & $\begin{array}{l}\text { No local } \\
\text { complications }\end{array}$ & $\begin{array}{l}\text { No } \\
\text { data* }\end{array}$ \\
\hline $\begin{array}{l}\text { Persistent organ } \\
\text { failure }\end{array}$ & 52 & 27 & 8 & 17 \\
\hline $\begin{array}{l}\text { Transient organ } \\
\text { failure }\end{array}$ & 27 & 7 & 17 & 3 \\
\hline No organ failure & 31 & 2 & 27 & 2 \\
\hline \multicolumn{5}{|c|}{$\begin{array}{l}\text { *No data from } 14 \text { patients that died in the first week and eight survivors } \\
\text { with inadequate records to assess local complications. } \\
\text { Local complications were necrosis or pseudocyst; early fluid collections } \\
\text { were not considered as complications. } \\
\text { Significant association of persistent organ failure and local complications: } \\
\chi^{2}=34.23 \text {; } d f=2 ; p<0.001 \text {. }\end{array}$} \\
\hline
\end{tabular}

not require routine follow up computed tomography (CT), and confirmation of the presence or absence of local complications was often lacking. Therefore, the relationship between early organ failure and subsequent local complications was examined in a subgroup of patients for whom a serious adverse event was recorded in the trial database. In these patients $(n=110)$ there were 14 early deaths within the first week. Of 36 patients who subsequently developed a local complication, 20 died, compared with five of 52 without documented local complications (missing data in eight survivors). There was a significant association between duration of early organ failure and later detection of local complications (table 5).

\section{DISCUSSION}

This study demonstrates that the duration of organ failure is a powerful marker of subsequent poor outcome in acute pancreatitis. The patient group studied was large, and was drawn from a wide range of hospitals. The findings therefore are likely to be representative of patients with predicted severe pancreatitis. The database used for this analysis has been reported previously. ${ }^{10}$ In that report, there was no overall difference in mortality rate between the treatment and placebo groups, so we feel it is unlikely that treatment given in the trial will have affected the present observations. We have included in the present report all patients initially diagnosed as predicted severe acute pancreatitis including those (three patients) who died and were found to have an incorrect diagnosis. This ensures that our findings accurately reflect the clinical situation.

We reiterate an important observation that selection of a group of patients with predicted severe acute pancreatitis using the APACHE-II score will identify many patients who already have organ failure. In this database, patients were selected with an APACHE-II score $>6$, according to the protocol of the previously described trial. ${ }^{10}$ Despite the lower threshold used here than the cut off level $(>8)$ suggested in the Atlanta criteria, ${ }^{5} 44 \%$ of patients had organ failure at entry to the study and over $50 \%$ had signs of organ failure within the first 24 hours. The proven value of APACHE-II as a predictor of severe acute pancreatitis ${ }^{13}$ probably relates to the ability of this system to identify early organ failure. Our data show that even with the lower cut off, there is a high subsequent rate of organ failure or local complications: 186 of $290(64 \%)$ had severe pancreatitis, as defined at Atlanta. Within this group of patients with predicted severe disease, the persistence of organ failure during the first week of hospital treatment is a powerful marker of fatal outcome. Mortality rate in patients with persistent organ failure was $35 \%$ (table 2 ), much greater than the mortality rate of $18 \%$ observed in patients with an initial APACHE-II score $>8$.
Although the observation that approximately half the patients with a raised APACHE-II score had organ failure in the first day or two of hospital admission limits the usefulness of systemic organ failure as a marker of response to treatment in randomised trials, it has been suggested ${ }^{6}$ that the progress of the organ failure itself could be a useful marker of disease severity. Buter and colleagues ${ }^{6}$ also used a cut off APACHE-II score $>6$ to predict severity and found that deterioration of organ failure was associated with a mortality rate greater than $50 \%$. They did not comment on the duration of early organ failure.

The present study clarifies the relationship between the dynamic nature of organ failure and subsequent death. Our data show that patients with organ failure which resolved within 48 hours have a very low risk of death, whereas organ failure which persists for longer than this time, even if not initially progressive, carries a grave prognosis.

The relationship between duration of organ failure and risk of death was similar, irrespective of the time of onset of organ failure. Although most patients who developed organ failure had done so at the time of study entry or shortly thereafter, 22 patients $(7.5 \%)$ developed new organ failure after the first 24 hours of the study. Of these, half had transient organ failure with no deaths and half $(\mathrm{n}=1 \mathrm{l})$ had persistent organ failure with four deaths. There was no difference in mortality rate between initial organ failure and new organ failure developing within the first week, suggesting that the important determinant of risk of death is the persistence of organ failure for more than 48 hours.

This analysis shows for the first time that persistence of organ failure during the first week is strongly associated with subsequent development of local complications. This is unsurprising given the association we observed of persistent organ failure with late deaths and the fact that most late deaths are associated with the presence of local complications. ${ }^{14}$ It suggests that the presence of extensive necrosis during the first week of the illness may be a factor in the persistence of organ failure but it does not exclude the opposite relationship as it is possible that persistent organ failure with associated poor oxygenation and reduced perfusion of the inflamed pancreas may contribute to extension of necrosis. The relationship between organ failure, necrosis, and fatal outcome is not well understood: Le Mee and colleagues $^{15}$ found a high mortality rate in infected necrosis, associated with multiple (three or more) organ failure; some authors found no correlation of organ failure with the extent of pancreatic necrosis or the presence of infection; ${ }^{16}{ }^{17}$ and in contrast, Lankisch and colleagues ${ }^{18}$ and Isenman and colleagues ${ }^{4}$ found organ failure more commonly in the presence of infection but in their patients the extent of necrosis was related to organ failure only in sterile necrosis. Lankisch and colleagues ${ }^{18}$ pointed out that initial organ failure and (more severe) late organ failure were both more common in patients with pancreatic necrosis but that both initial and late organ failure, and pancreatic necrosis, could occur independently in many patients. The present data suggest that persistent organ failure during the first week of admission is a marker of increased risk of pancreatic necrosis, as manifest in local complications, but the data do not clarify whether the association depends on necrosis causing organ failure or organ failure contributing to the incidence and extent of pancreatic necrosis.

In this study there was no systematic search for local complications. CT was not employed routinely during the first week of the illness, nor was a follow up CT scan required by the trial protocol. Consequently, there may be some underreporting of local complications. However, all patients were followed until discharge from hospital, and any patient readmitted with a pancreatitis related problem was 
documented. Therefore, we are confident that the large majority of symptomatic local complications have been recorded. It can be argued that asymptomatic areas of necrosis are not clinically significant. In the subgroup of patients with documented serious adverse events, there was a clear relationship between persistence of early organ failure and late development of local complications (table 5). This study suggests that persistent organ failure in the first week could be a useful marker for the subsequent development of symptomatic local complications.

We have not commented on the severity of organ failure, which has been shown by others to be associated with local complications. ${ }^{15}$ Instead, we chose to apply the Atlanta criteria ${ }^{5}$ which depend on whether the threshold for organ failure has been passed. Transient organ failure was associated with a good outcome; most of these patients had single organ failure. However, persistent organ failure identifies patients at increased risk of death, and although most patients who died had multiple organ failure in the first week of their illness, late death was associated in more than $25 \%$ of cases with persistent single organ failure during the first week.

Patients with transient organ failure almost always had single organ failure, usually pulmonary. Only four patients with transient organ failure had two organ failures. This is further supporting evidence that transient organ failure is associated with less severe disease as the incidence of multiple organ failure is much lower than in patients with persistent organ failure.

This analysis cannot shed light on whether the dynamic nature of organ failure can be modified with a beneficial effect on outcome. We have observed a marker of poor outcome (persistent organ failure). It is tempting to speculate that efforts to ameliorate organ failure early in the course of acute pancreatitis might improve outcome. If the persistence of organ failure leads to systemic hypoxia, and perhaps reduced perfusion, that could be a mechanism to cause or extend areas of necrosis in the pancreas. In that case, early aggressive circulatory support with intravenous fluids and inotropes if necessary, and oxygen supplementation, might lead to improved pancreatic perfusion and a reduced risk of necrosis and subsequently death. Experimental evidence suggests that disturbance of the microcirculation may contribute to the development of pancreatic necrosis. ${ }^{19-21}$ There is some clinical evidence that early aggressive resuscitation may improve outcome ${ }^{22} 23$ which lends support to this hypothesis.

However, there is probably an irreversible minimum mortality in severe acute pancreatitis, dependent on the severity of the early organ dysfunction syndrome. A substantial minority of our patients died within the first week of the illness of rapidly progressive multiple organ failure. Until an effective mechanism is found to interrupt the downward spiral of accelerating organ failure in these patients, general supportive care is all that can be offered. Nevertheless, these findings suggest the tantalising possibility that patients with persistent early organ failure represent a group at high risk of local complications and late death in whom aggressive supportive therapy and appropriate management of pancreatic necrosis might have the potential to improve outcome.

The importance of this study to our understanding of the pathophysiology of acute pancreatitis lies in the recognition that a single record of organ failure, as defined in the Atlanta criteria, ${ }^{5}$ is insufficient for the diagnosis of severe acute pancreatitis. Patients without early organ failure, or with early organ failure that resolves within 48 hours, are at very low risk of developing local complications and death. This calls into question whether such transient organ failure should be reckoned as a complication of pancreatitis and a marker of severe disease. Such patients usually respond rapidly to initial therapy and most leave the hospital within seven days.

Selection of patients for clinical trials of therapy is difficult in acute pancreatitis. Currently, APACHE-II is widely used to identify patients at high risk of complications although this system is not ideal and efforts continue to improve prediction using other features, such as obesity. ${ }^{24}$ As noted above, APACHE-II identifies a group of patients in which a majority already have organ failure. This is unsatisfactory if the presence of complications is used as an end point as therapy cannot influence the high pre-existing rate of organ failure. In the present study, an APACHE-II score $>8$ identified almost all patients who died from pancreatitis but selected a group with only half the mortality rate of that seen in patients with persistent early organ failure. If death or local complications are to be the end point of a clinical trial, persistent organ failure in the first week of hospital admission seems to be an appropriate way to select patients for study.

In conclusion, we have extended the observation of Buter and colleagues ${ }^{6}$ that the dynamic nature of organ failure arising during the first week of an attack of acute pancreatitis has a substantial prognostic effect. Patients with organ failure that resolves within 48 hours have a very low risk of local complications and death. In contrast, patients who have organ failure (as defined in the Atlanta ${ }^{5}$ criteria, equivalent to any organ score of two or more in the Marshall system ${ }^{12}$ ) which persists for three or more days have a greater than one in three risk of fatal outcome. Local complications arise in the majority of patients with persistent organ failure. In contrast, transient organ failure has only a $25 \%$ risk of symptomatic local complications and these patients rarely die.

\section{Authors' affiliations \\ C D Johnson, M Abu-Hilal, University Surgical Unit, Southampton \\ General Hospital, Southampton, UK}

\section{APPENDIX}

\section{MEMBERS OF THE BRITISH ACUTE PANCREATITIS STUDY GROUP}

Professor D Alderson, Bristol Royal Infirmary; Mr A Berry, Northampton General Hospital; Dr D Breen, Royal Hallamshire Hospital, Sheffield; Mr J Britton, John Radcliffe Hospital, Oxford; Mr C Brown, Derriford Hospital, Plymouth; Professor FC Campbell, Ninewells Hospital, Dundee and The Medical School, Newcastle; Mr R Edmonson, Lewisham Hospital; Mr K Fearon, Edinburgh Royal Infirmary; Dr C Garrard, John Radcliffe Hospital, Oxford; Professor CW Imrie, Glasgow Royal Infirmary; Professor A Kingsnorth, Royal Liverpool Hospital and Derriford Hospital, Plymouth; Mr M Larvin, Leeds General Infirmary; Mr DM Lloyd, Leicester Royal Infirmary; Mr C McKay, Glasgow Royal Infirmary; Professor MJ McMahon, Leeds General Infirmary; Professor JP Neoptolemos, Queen Elizabeth Hospital, Birmingham; Professor BJ Rowlands, Royal Victoria Hospital, Belfast; Mr S Shimi, Ninewells Hospital, Dundee; Mr R Sutton, Royal Liverpool Hospital; Dr D Thomas, Morriston Hospital, Swansea; Mr RA L Young, West Middlesex University Hospital.

\section{REFERENCES}

1 McKay CJ, Evans S, Sinclair $M$, et al. High early mortality rate from acute pancreatitis in Scotland, 1984-1995. Br J Surg 1999;86:1302-5. 
2 Toh SK, Phillips S, Johnson CD. A prospective audit against national standards of the presentation and management of acute pancreatitis in the South of England. Gut 2000;46:239-43.

3 Ashley SW, Perez A, Pierce EA, et al. Necrotizing pancreatitis: contemporary analysis of 99 consecutive cases. Ann Surg 2001;234:572-9.

4 Isenmann R, Rau B, Beger HG. Bacterial infection and extent of necrosis are determinants of organ failure in patients with acute necrotizing pancreatitis. Br J Surg 1999;86:1020-4.

5 Bradley EL III. A clinically based classification system for acute pancreatitis. Summary of the International Symposium on Acute Pancreatitis, Atlanta, Ga, September 11 through 13, 1992. Arch Surg 1993;128:586-90

6 Buter A, Imrie CW, Carter CR, et al. Dynamic nature of early organ dysfunction determines outcome in acute pancreatitis. $\mathrm{Br} J$ Surg 2002;89:298-302.

7 United Kingdom guidelines for the management of acute pancreatitis. British Society of Gastroenterology. Gut 1998;42(suppl 2):S1-13.

8 Beger HG, Bittner R, Block S, et al. Bacterial contamination of pancreatic necrosis. A prospective clinical study. Gastroenterology 1986;91:433-8.

9 Buchler MW, Gloor B, Muller CA, et al. Acute necrotizing pancreatitis: treatment strategy according to the status of infection. Ann Surg 2000;232:619-26.

10 Johnson CD, Kingsnorth AN, Imrie CW, et al. Double blind, randomised, placebo controlled study of a platelet activating factor antagonist, lexipafant, in the treatment and prevention of organ failure in predicted severe acute pancreatitis. Gut 2001;48:62-9.

11 Knaus WA, Draper EA, Wagner DP, et al. APACHE II: a severity of disease classification system. Crit Care Med 1985;13:818-29.

12 Marshall JC, Cook DJ, Christou NV, et al. Multiple organ dysfunction score: a reliable descriptor of a complex clinical outcome. Crit Care Med 1995;23:1638-52.

13 Larvin M, McMahon MJ. APACHE-II score for assessment and monitoring of acute pancreatitis. Lancet 1989;2:201-5.
14 Blum T, Maisonneuve $P$, Lowenfels $A B$, et al. Fatal outcome in acute pancreatitis: its occurrence and early prediction. Pancreatology 2001;1:237-41.

15 Le Mee J, Paye F, Sauvanet A, et al. Incidence and reversibility of organ failure in the course of sterile or infected necrotizing pancreatitis. Arch Surg 2001; 136:1386-90

16 Tenner S, Sica G, Hughes $M$, et al. Relationship of necrosis to organ failure in severe acute pancreatitis. Gastroenterology 1997;113:899-903.

17 Zhu AJ, Shi JS, Sun XJ. Organ failure associated with severe acute pancreatitis. World J Gastroenterol 2003;9:2570-3.

18 Lankisch PG, Pflichthofer D, Lehnick D. No strict correlation between necrosis and organ failure in acute pancreatitis. Pancreas 2000;20. 319-22.

19 Klar E, Messmer K, Warshaw AL, et al. Pancreatic ischaemia in experimental acute pancreatitis: mechanism, significance and therapy. Br J Surg 1990;77:1205-10.

20 Klar E, Rattner DW, Compton C, et al. Adverse effect of therapeutic vasoconstrictors in experimental acute pancreatitis. Ann Surg 1991;214:168-74.

21 Werner J, Z'graggen K, Fernandez-del Castillo C, et al. Specific therapy for local and systemic complications of acute pancreatitis with monoclonal antibodies against ICAM-1. Ann Surg 1999;229:834-40.

22 Klar E, Foitzik T, Buhr H, et al. Isovolemic hemodilution with dextran 60 as treatment of pancreatic ischemia in acute pancreatitis. Clinical practicability of an experimental concept. Ann Surg 1993;217:369-74.

23 Brown A, Baillargeon JD, Hughes MD, et al. Can fluid resuscitation prevent pancreatic necrosis in severe acute pancreatitis? Pancreatology 2002; 2:104-7.

24 Johnson CD, Toh SKC, Campbell MJ. Combination of APACHE-II score and an obesity score (APACHE-O) for the prediction of severe acute pancreatitis. Pancreatology 2004; 4: 1-6.

\section{EDITOR'S QUIZ: GI SNAPSHOT}

\section{Painful ascites}

\section{Clinical presentation}

A 67 year old woman presented initially with a history of recurrent urinary tract infections. Routine imaging with ultrasound incidentally detected a $7 \mathrm{~cm} \times 8 \mathrm{~cm}$ well defined echogenic mass within the right lobe of the liver. Follow up with six monthly imaging was planned. Five months after initial presentation, the patient presented with abdominal distension and pain. Clinical examination revealed icterus, hepatomegaly, and ascites. Blood parameters were as follows: bilirubin $51 \mu \mathrm{mol} / \mathrm{l}$, alanine aminotransferase $160 \mathrm{IU} / \mathrm{l}$, alkaline phosphatase $294 \mathrm{IU} / \mathrm{l}$, gamma glutamyl transferase $136 \mathrm{IU} / \mathrm{l}$, albumin $37 \mathrm{mg} / \mathrm{dl}$, haemoglobin $15.8 \mathrm{~g} / \mathrm{dl}$, WCC $8.6 \times 10^{9} /$, platelets $337 \times 10^{9} / \mathrm{l}$, prothrombin time 14.8 seconds, and $\alpha$ fetoprotein $3.1 \mathrm{IU} / \mathrm{l}$. A contrast enhanced computerised tomography scan showed a $8 \times 7 \mathrm{~cm}$ mass of predominantly fatty attenuation, caudate lobe hypertrophy, and ascites. Gadolinium enhanced magnetic resonance imaging showed that the mass was composed of fat and vascular tissue.

\section{Question}

What conclusions could be drawn from this image (fig 1)?

See page 1355 for answer

This case is submitted by:

S Sebastian, D Tuite, P Crotty, W Torreggiani, M J Buckley Adelaide and Meath Hospital, Dublin, Ireland

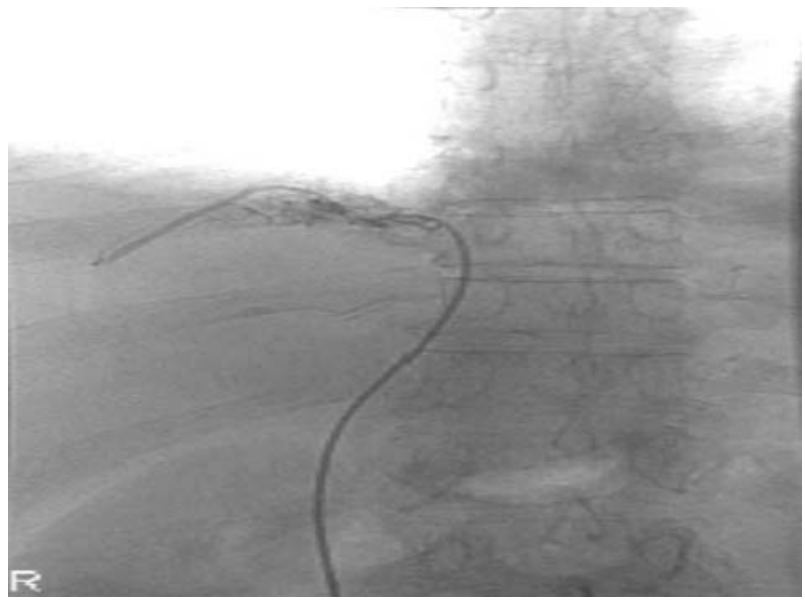

Figure 1 Hepatic venography showing absence of flow in the hepatic veins

Correspondence to: Dr Shaji Sebastian Department of Gastroenterology, Adelaide and Meath Hospital, Tallaght, Dublin 24, Ireland sebgastro@eircom.net

doi: 10.1136/gut.2003.031609 